\title{
THE EFFECT OF HEAT AND AMMONIA TREATMENT ON COLOUR RESPONSE OF OAK WOOD (Quercus robur) AND COMPARISON OF SOME PHYSICAL AND MECHANICAL PROPERTIES
}

\author{
Petr Čermák ${ }^{1, \$}$, Aleš Dejmal $^{1}$
}

\begin{abstract}
In this paper the effect of heat and ammonia treatment on the change in colour of oak wood (Quercus robur) and comparison of physical and mechanical properties were investigated. Wood specimens were made from heartwood and sapwood in dimensions $30 \times 30 \times 30 \mathrm{~mm}^{3}$. The specimens were subjected to heat treatment at $180^{\circ} \mathrm{C}$ and $230^{\circ} \mathrm{C}$ for 2 and 4 hours, others were treated by ammonia for 24 hours. After these processes, CIELAB colour, hardness, EMC, density and MOE of wood were tested in comparison with untreated samples. The results show that the heat treatment contributed to darkening of sapwood and heartwood and the total colour change increased up to 38.47 units (CIELAB) in the radial direction and 37.75 units (CIELAB) in the tangential direction. Previous research into ammonia treatment shows that the most significant changes were noticed after 1 day. The colour changes between sapwood and heartwood were more noticeable in ammonia treatment than in the case of heat treatment. All other observed properties were decreased in the case of heat treatment. Ammonia treatment does not have a significant influence on the other observed properties. The results proved that ammonia treatment is a modification that improves the decorative value but has a minimal effect on technologically relevant properties of solid wood. Nevertheless, the heat treatment can be used as comparable process for colour change of wood.
\end{abstract}

Keywords: Ammonia treatment, CIELAB, colour change, density, equilibrium moisture content, Janka hardness, heat treatment, modulus of elasticity, Quercus $s p$.

\section{INTRODUCTION}

Wood is a complex and natural material that consists of cellulose (35-55\%), hemicelluloses (20-35\%) and lignin (15-36\%) and other inorganic and organic compounds (3-10\%). Cellulose represents the crystalline area of the wood, hemicelluloses and lignin make up the amorphous area. These compounds constitute the colour of wood. The colour can be changed by many processes, for example heat and ammonia treatment.

Heat treatment has important effects on the colour and the chemical composition of the wood. Colour is a very important wood property for the final use, and in some cases it is the determining factor for the selection of a specific wood. Most wood colour studies quantify the colour by the CIELAB method with a three axes system (Brischke et al. 2007, Baar and Gryc 2012, Bekhta and Niemz 2003). The specimens become darker with an increasing treatment time and temperature as reported by several authors (Mitsui et al. 2001, 2003, 2004; Militz 2002, Bekhta and Niemz 2003). Esteves et al. (2008) reported that noticeable colour changes could already be obtained for small mass losses of $2-4 \%$, but the effect depended on the extent of treatment and was related to the chemical composition of the heat treated woods. The darker tonality of heat treated wood is often attributed to the formation of coloured degradation products from hemicelluloses (Sehlstedt-Persson 2003, Sundqvist 2004) and to extractives that seem to participate in the colour formation of heat treated wood (McDonald et al. 1997, Sundqvist and Morén 2002). The main effect of the heat treatment is the decrease in equilibrium moisture content (EMC). The reduction was already reported in 1920 by Tiemann, who showed that drying at high temperatures decreased the equilibrium moisture of wood and consequently it is swelling and shrinking. According to Jämsä and Viitaniemi (2001), the reason for the decrease in the equilibrium moisture content is that less water is absorbed by the cell walls after the heat treatment

${ }^{1}$ Department of Wood Science, Mendel University in Brno, 61300 Brno, Czech Republic

^Corresponding author: xcerma24@node.mendelu.cz

Received: 21.11.2012. Accepted: 24.03.2013. 
as a result of the chemical change with a decrease in hydroxyl groups. Boonstra et al. (2007) and Korkut et al. (2008) found that the Brinell hardness parallel to the grain increased significantly (48\%), whereas the hardness perpendicular to the grain increased slightly (5\%). One of the main heat treated wood limitations is the decrease in mechanical strength. The first tests made by Stamm et al. (1946) showed that MOE increased slightly for wood treated at $180^{\circ} \mathrm{C}$ and $200^{\circ} \mathrm{C}$, decreasing afterwards, reaching a $10 \%$ decrease after 20 hours at $180^{\circ} \mathrm{C}$ and 8 hours at $200^{\circ} \mathrm{C}$. With the treatment at $220^{\circ} \mathrm{C}$, MOE decreased drastically by about $60 \%$ in only 7 hours. Vavrčík et al. (2008) reported the average density of oak wood (Quercus robur) as $618.2 \mathrm{~kg} \mathrm{~m}^{-3}$. Similar results were obtained by Vavrčík and Gryc (2012), which presented average density $584.3 \mathrm{~kg} \mathrm{~m}^{-3}$. Also was confirmed the influence of stand and position in the trunk. The density of wood treated between $200^{\circ} \mathrm{C}$ and $260^{\circ} \mathrm{C}$ decreased from $447 \mathrm{~kg} \mathrm{~m}^{-3}$ to 381 $\mathrm{kg} \mathrm{m}^{-3}$ and from $623 \mathrm{~kg} \mathrm{~m}^{-3}$ to $617 \mathrm{~kg} \mathrm{~m}^{-3}$ for spruce and beech wood, respectively, corresponding to a decrease of $15 \%$ and $1 \%$. Boonstra et al. (2007) also reported a $10 \%$ and $8.5 \%$ decrease in density for heat treated Scots pine and Norway spruce, respectively.

The ammonia treatment as a method to change the colour of wood is well known and has been explored by several authors (Tinkler 1921, Bariska 1969). Weigl et al. (2007, 2009a, b) showed the effect of ammoniation on the colour of wood. With proper treatment light-coloured wood is darkened and may imitate exclusive timber (Weigl et al. 2009b). Ammonia treated wood is therefore frequently applied in interior design for furniture or flooring. The most often used species for ammonia treatment is oak. Colour changes of many different wood species following ammonia treatment procedure have been studied (Weigl et al. 2009c), and values of total colour changes were found to vary between 8 and 42. According to Tinkler (1921), changes in colour are due to the reaction of ammonia with tannin in the wood and atmospheric oxygen. Sapwood of oak contains just a little of tannin, due to which the colour changes is smaller. Significant changes in EMC due to the ammonia treatment process were found by Weigl et al. (2012), which was consistent with earlier findings (Weigl et al. 2009c). The observed magnitude is not of practical relevance in the case of oak (an average increase of $0.5 \%$ ), and the same is true for the two softwood (Norway spruce and Scots pine) species showing even lower differences compared to oak. No significant changes in wood density were found using ammonia treatment for any of the investigated species (Weigl et al. 2012). That is why EMC and density variations seem to be irrelevant with respect to the possible impact on mechanical properties of ammonia treated wood. Significant changes in MOE were partly observed for oak wood. However, stiffness and strength losses were in the range of 6 to $15 \%$, which is negligible with respect to common solid wood applications.

This study deals with the effect of heat and ammonia treatment on solid wood properties. The main aim of this paper is to compare the influence of these processes on colour response and the extent of the changes of physical and mechanical properties.

\section{MATERIAL AND METHODS}

Wood specimens used for this study were obtained from oak wood (Quercus robur). Specimens with dimensions $30 \times 30 \times 30 \mathrm{~mm}^{3}$ in tangential, radial and longitudinal directions were used. Half of them were taken from heartwood, the other half from sapwood. Samples were stored at conditions $\mathrm{T}=20^{\circ} \mathrm{C} ; \mathrm{RH}=40 \%$ until equilibrium moisture content (EMC) was reached $-7.5 \%$. The references values of colour tone and hardness were measured before treatment.

The specimens were divided into sets of 30 samples for each material (sap- and heartwood) and each treatment time and temperature (Table 1). Time of ammonia treatment was chosen based on a previous test. It shows that the most significant colour change occurred during the first day. Heat treatment was performed using a universal high-temperature box SIEMENS HB36D.70. The used procedure was by ThermoWood technology, developed by VTT in Finland. Specimens were exposed to ammonia for $24 \mathrm{~h}$. The ammonia treatment was performed at a temperature of $20^{\circ} \mathrm{C}$ under atmospheric pressure in a closed fume cupboard. Samples were placed on plastic grids above the open surface of ammonia solution with concentration $25 \%(0,3 \mathrm{ml}$ of $25 \%$ solution per $1 \mathrm{~g}$ of wood). 
Table 1. Sets of 30 samples for each material and each treatment.

\begin{tabular}{cccc}
\hline Material & $\begin{array}{c}\text { Temperatures } \\
\left({ }^{\circ} \mathrm{C}\right)\end{array}$ & Treatment & $\begin{array}{c}\text { Times } \\
(\mathrm{h})\end{array}$ \\
\hline Oak sapwood & $180 ; 230$ & heat & $2 ; 4$ \\
Oak heartwood & $180 ; 230$ & heat & $2 ; 4$ \\
Oak sapwood & & ammonia & 24 \\
Oak heartwood & & ammonia & 24 \\
Oak sap/heartwood & reference & untreated & \\
\hline
\end{tabular}

Colour measurements of all specimens were taken on tangential and radial surfaces of the wood specimens before and after treatments by a spectrophotometer BYK spectro-guide 45/0 in colour system CIELAB. The diameter of sensor head was $11 \mathrm{~mm}$. Measurements were made using a 10-degree standard observer and D 65 illuminant. Colour system CIELAB is defined as follows: $\mathrm{L}^{*}$ is the value of lightness, $\mathrm{a}^{*}$ is the chroma from green to red and $\mathrm{b}^{*}$ is the chroma from blue to yellow. From these values the differences $\left(\Delta \mathrm{L}^{*}, \Delta \mathrm{a}^{*}\right.$ and $\left.\Delta \mathrm{b}^{*}\right)$ can be calculated as well as the total colour difference $\left(\Delta \mathrm{E}^{*}\right)$ between treated and untreated wood.

$$
\Delta E^{*}=\sqrt{\left(\Delta L^{*}\right)^{2}+\left(\Delta a^{*}\right)^{2}+\left(\Delta b^{*}\right)^{2}}
$$

The measurements of hardness and modulus of elasticity in compression were performed on Zwick 050 machine by the Janka method. Janka hardness test measures the resistance of a type of wood to denting and wear. It measures the force required to embed an $11.28 \mathrm{~mm}$ steel ball into wood. The values of modulus of elasticity were calculated by software testXpert, hardness was calculated by the following equation.

$$
H_{j}=4 F / 3 \pi r^{2}
$$

Density was measured by common equation (Siau 1984). Equilibrium moisture content was observed on all specimens which were subjected to the same conditions as the untreated ones.

\section{RESULTS AND DISCUSSION}

\section{The effect of heat and ammonia treatment on wood colour change}

The following graphs show the influence of lightness $\left(\mathrm{L}^{*}\right)$, chroma from green to red $\left(\mathrm{a}^{*}\right)$ and chroma from blue to yellow $\left(b^{*}\right)$ on the final colour change $\left(\Delta \mathrm{E}^{*}\right)$. All results are shown for the radial as well as for the tangential surface. In all graphs is also added information about tangential surface in the middle of thickness (testing samples were half-splitted). 


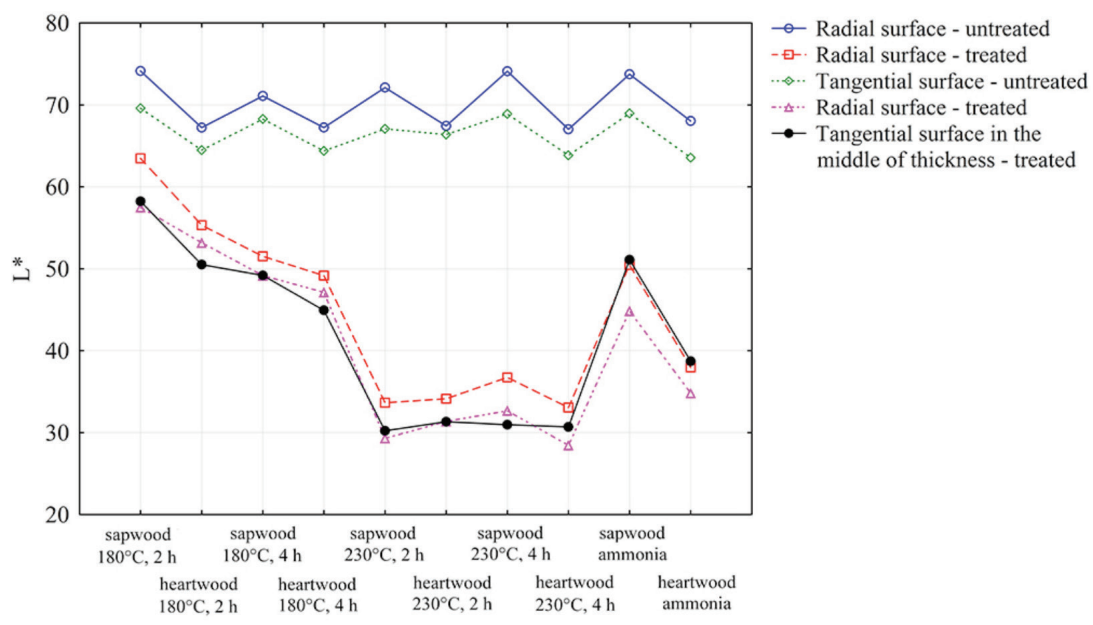

Figure 1. Changes of lightness ( $\left.\mathrm{L}^{*}\right)$ for all treated and untreated specimens.

Figure 1 depicts an influence of treatment, temperature and time on lightness $\left(\mathrm{L}^{*}\right)$. This factor most contributed to darkening of specimens. The most significant changes were observed after heat treatment at $230^{\circ} \mathrm{C}$ on average by 36 units. Bekhta and Niemz (2003) also studied effect of heat treatment on colour response. This work mentioned decrease about 30 units at temperature $200^{\circ} \mathrm{C}$ for 2 hours respectively 40 units at temperature $200^{\circ} \mathrm{C}$ for 4 hours. Important changes can be seen for ammonia treatment as well. The differences between sap- and heartwood were more noticeable. Heartwood became by about 6.8 units darker on the radial surface and 4.6 units on the tangential surface more than sapwood. $\Delta \mathrm{L}^{*}$ value 25 units due ammonia treatment corresponding to Weigl et al. (2012), which mentioned values 17, 22 and 26 for spruce, oak and pine, respectively.

Figure 2 shows an influence of treatment, temperature and time on chroma from red to green $\left(a^{*}\right)$. The changes of this factor are quite small - up to 2 units. A slight increase in this factor can be noticed inside of specimens treated by heat at $230^{\circ} \mathrm{C}$ or by ammonia. Weigl et al. (2012) also observed 2 units difference after ammonia treatment. Also Bekhta and Niemz (2003) show increase about 3-4 units.

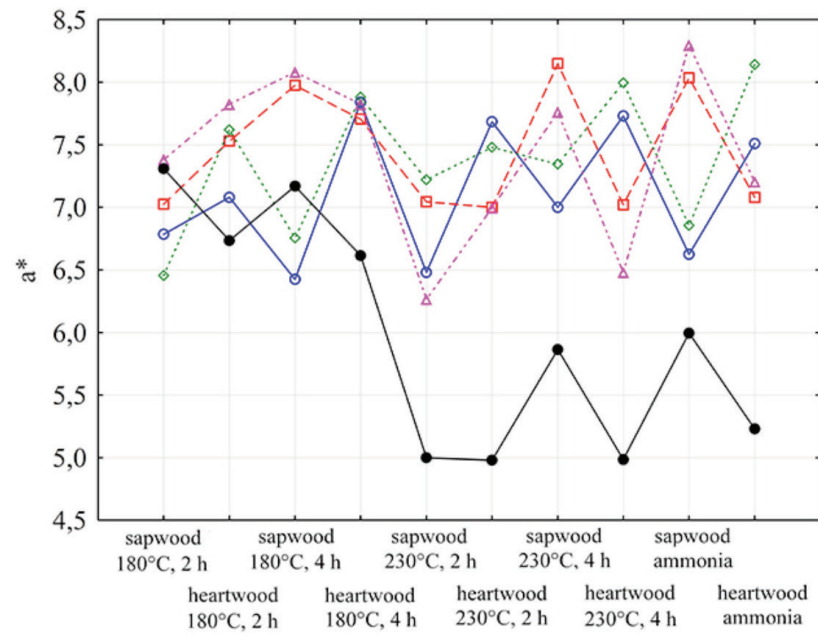

$\rightarrow$ Radial surface - untreated

- $\bullet-$ Radial surface - treated

$\cdots \otimes$ Tangential surface - untreated

$\cdots$ Radial surface - treated

$\rightarrow$ Tangential surface in the middle of thickness - treated

Figure 2. Changes of chroma from green to red $\left(\mathrm{a}^{*}\right)$ for all treated and untreated specimens. 
Figure 3 depicts an influence of treatment, temperature and time on chroma from blue to yellow ( $\left.b^{*}\right)$. This factor was more significant than $\mathrm{a}^{*}$. At temperature $180^{\circ} \mathrm{C}$ a decrease in yellows tone was noticed on surface by about 3.4 units, while at $230^{\circ} \mathrm{C}$ by up to 10.4 units. Sapwood treated by ammonia turned to yellows tone by about 0.5 units, while heartwood turned to blue tone by about 6.7 units on the radial and 5.7 units on tangential surface as mentioned by Weigl et al. (2012) which presented values 8 for oak wood. Similar values of ( $\left.b^{*}\right)$ also presented Bekhta and Niemz (2003), increase about 5-10 units.

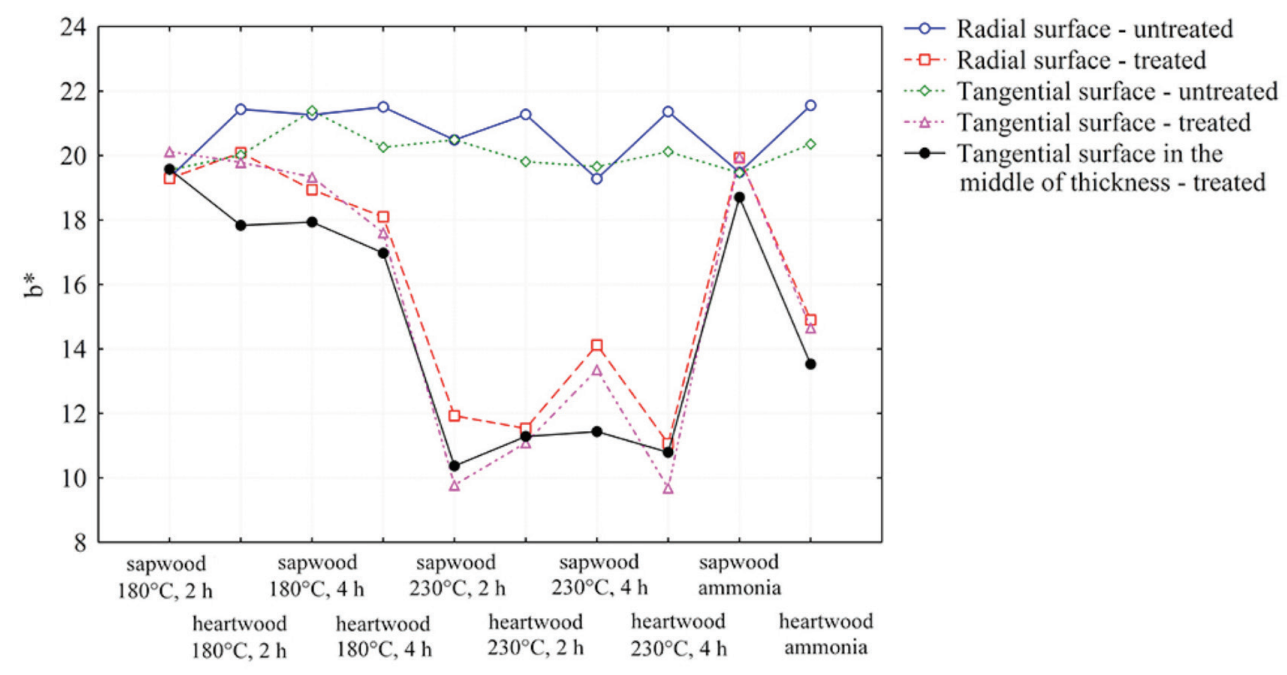

Figure 3. Changes of chroma from blue to yellow $\left(b^{*}\right)$ for all treated and untreated specimens.

Table 2 shows basic statistic values of total colour changes $\left(\Delta E^{*}\right)$ for all investigated conditions. The last column of table assess whether data came from a normal distribution or not. 
Table 2. Basic statistic values of total colour changes $\left(\Delta \mathrm{E}^{*}\right)$ for all conditions.

\begin{tabular}{|c|c|c|c|c|c|c|c|c|c|c|}
\hline & 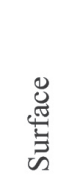 & $\begin{array}{l}\frac{n}{2} \\
\frac{0}{\pi} \\
\frac{\pi}{0} \\
\dot{0} \\
\dot{z}\end{array}$ & $\begin{array}{l}0 \\
50 \\
\pi \\
\frac{\pi}{2} \\
2 \\
2\end{array}$ & 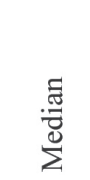 & $\stackrel{\Xi}{\Xi}$ & 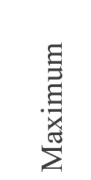 & 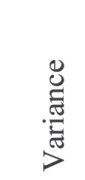 & 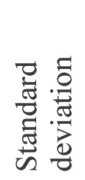 & 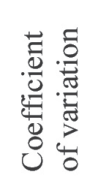 & $\begin{array}{l}\text { 营 } \\
\text { : } \\
\text { Z }\end{array}$ \\
\hline \multirow[t]{2}{*}{ Sapwood $180^{\circ} \mathrm{C} 2 \mathrm{~h}$} & $\overline{T T}$ & 30 & 10,77 & 10,89 & 0,49 & 13,42 & 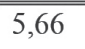 & 2,38 & 22,10 & NO \\
\hline & $\mathrm{R}$ & 30 & 13,34 & 12,20 & 8,23 & 24,16 & 12,89 & 3,59 & 26,91 & NO \\
\hline \multirow[t]{2}{*}{ Sapwood $180^{\circ} \mathrm{C} 4 \mathrm{~h}$} & $\mathrm{~T}$ & 30 & 20,90 & 18,88 & 12,13 & 38,73 & 47,56 & 6,90 & 32,99 & NO \\
\hline & $\mathrm{R}$ & 30 & 21,56 & 21,41 & 13,30 & 40,54 & 36,24 & 6,02 & 27,92 & NO \\
\hline \multirow[t]{2}{*}{ Sapwood $230^{\circ} \mathrm{C} 2 \mathrm{~h}$} & $\mathrm{~T}$ & 30 & 38,80 & 36,73 & 29,61 & 47,90 & 26,79 & 5,18 & 13,34 & $\mathrm{NO}$ \\
\hline & $\mathrm{R}$ & 30 & 40,28 & 40,18 & 30,86 & 49,67 & 23,35 & 4,83 & 11,99 & YES \\
\hline \multirow[t]{2}{*}{ Sapwood $230^{\circ} \mathrm{C} 4 \mathrm{~h}$} & $\mathrm{~T}$ & 30 & 37,62 & 37,28 & 31,53 & 44,12 & 10,49 & 3,24 & 8,61 & YES \\
\hline & $\mathrm{R}$ & 30 & 37,00 & 36,62 & 26,33 & 47,65 & 14,82 & 3,85 & 10,40 & YES \\
\hline \multirow[t]{2}{*}{ Sapwood ammonia } & $\mathrm{T}$ & 30 & 24,89 & 23,08 & 16,13 & 39,56 & 39,65 & 6,30 & 25,29 & NO \\
\hline & $\mathrm{R}$ & 30 & 25,61 & 24,25 & 14,46 & 48,73 & 61,22 & 7,82 & 30,56 & $\mathrm{NO}$ \\
\hline \multirow[t]{2}{*}{ Heartwood $180^{\circ} \mathrm{C} 2 \mathrm{~h}$} & $\mathrm{~T}$ & 30 & 12,05 & 11,46 & 9,13 & 18,21 & 5,53 & 2,35 & 19,51 & $\mathrm{NO}$ \\
\hline & $\mathrm{R}$ & 30 & 11,56 & 11,58 & 8,20 & 17,02 & 6,72 & 2,59 & 22,43 & $\mathrm{NO}$ \\
\hline \multirow[t]{2}{*}{ Heartwood $180^{\circ} \mathrm{C} 4 \mathrm{~h}$} & $\mathrm{~T}$ & 30 & 17,79 & 17,82 & 13,70 & 21,61 & 4,65 & 2,16 & 12,13 & YES \\
\hline & $\mathrm{R}$ & 30 & 18,68 & 18,70 & 13,55 & 34,50 & 15,43 & 3,93 & 21,02 & $\mathrm{NO}$ \\
\hline \multirow[t]{2}{*}{ Heartwood $230^{\circ} \mathrm{C} 2 \mathrm{~h}$} & $\mathrm{~T}$ & 30 & 35,07 & 34,38 & 31,59 & 40,83 & 4,64 & 2,15 & 6,14 & NO \\
\hline & $\mathrm{R}$ & 30 & 35,60 & 35,60 & 20,43 & 46,34 & 24,06 & 4,91 & 13,78 & YES \\
\hline \multirow[t]{2}{*}{ Heartwood $230^{\circ} \mathrm{C} 4 \mathrm{~h}$} & $\mathrm{~T}$ & 30 & 35,90 & 35,59 & 26,89 & 46,88 & 12,23 & 3,50 & 9,74 & $\mathrm{NO}$ \\
\hline & $\mathrm{R}$ & 30 & 37,04 & 36,44 & 30,59 & 47,54 & 16,54 & 4,07 & 10,98 & $\mathrm{NO}$ \\
\hline \multirow[t]{2}{*}{ Heartwood ammonia } & $\mathrm{T}$ & 30 & 32,82 & 31,11 & 21,97 & 52,32 & 53,71 & 7,33 & 22,33 & $\mathrm{NO}$ \\
\hline & $\mathrm{R}$ & 30 & 30,64 & 29,18 & 18,66 & 44,92 & 42,28 & 6,50 & 21,22 & YES \\
\hline
\end{tabular}

Table 3 y 4 depicts the evaluation of total colour changes by Krukal-Wallis test for the radial and tangential surface. After comparison of the total colour changes between sap and heartwood, treated at the same conditions, no significant statistical differences were observed. It means that the colour unification of sap and heartwood was not noticeable. Result from the radial surface and the tangential surface are almost the same. 
Table 3. Evaluation of total colour changes $\left(\Delta \mathrm{E}^{*}\right)$ by Kruskal-Wallis test - radial surface.

\begin{tabular}{|c|c|c|c|c|c|c|c|c|c|c|}
\hline \multicolumn{11}{|c|}{ Final evaluation of colour - total colour change $\left(\Delta \mathrm{E}^{*}\right)$} \\
\hline \multicolumn{5}{|l|}{ Kruskal-Wallis test } & \multicolumn{6}{|c|}{ RADIAL SURFACE } \\
\hline \multicolumn{4}{|l|}{ Established difference of data } & \multirow[t]{2}{*}{ YES } & & & & & & \\
\hline \multicolumn{10}{|l|}{ Multiple comparisons } & \\
\hline & 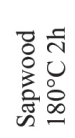 & 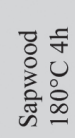 & 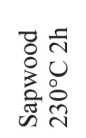 & 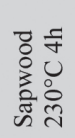 & 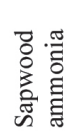 & 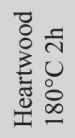 & 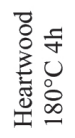 & 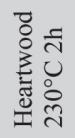 & 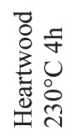 & 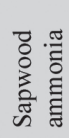 \\
\hline Sapwood $180^{\circ} \mathrm{C} 2 \mathrm{~h}$ & & Agr. & Dif. & Dif. & Agr. & Agr. & Agr. & Dif. & Dif. & Dif. \\
\hline Sapwood $180^{\circ} \mathrm{C} 4 \mathrm{~h}$ & Agr. & & Dif. & Dif. & Agr. & Agr. & Agr. & Dif. & Dif. & Dif. \\
\hline Sapwood $230^{\circ} \mathrm{C} 2 \mathrm{~h}$ & Dif. & Dif. & & Agr. & Dif. & Dif. & Dif. & Agr. & Agr. & Agr. \\
\hline Sapwood $230^{\circ} \mathrm{C} 4 \mathrm{~h}$ & Dif. & Dif. & Agr. & & Dif. & Dif. & Dif. & Agr. & Agr. & Agr. \\
\hline Sapwood ammonia & Agr. & Agr. & Dif. & Dif. & & Dif. & Agr. & Dif. & Dif. & Agr. \\
\hline Heartwood $180^{\circ} \mathrm{C} 2 \mathrm{~h}$ & Agr. & Agr. & Dif. & Dif. & Dif. & & Agr. & Dif. & Dif. & Dif. \\
\hline Heartwood $180^{\circ} \mathrm{C} 4 \mathrm{~h}$ & Agr. & Agr. & Dif. & Dif. & Agr. & Agr. & & Dif. & Dif. & Dif. \\
\hline Heartwood $230^{\circ} \mathrm{C} 2 \mathrm{~h}$ & Dif. & Dif. & Agr. & Agr. & Dif. & Dif. & Dif. & & Agr. & Agr. \\
\hline Heartwood $230^{\circ} \mathrm{C} 4 \mathrm{~h}$ & Dif. & Dif. & Agr. & Agr. & Dif. & Dif. & Dif. & Agr. & & Agr. \\
\hline Heartwood ammonia & Dif. & Dif. & Agr. & Agr. & Agr. & Dif. & Dif. & Agr. & Agr. & \\
\hline
\end{tabular}

Agr. - Agreement

Dif. - Difference 
Table 4. Evaluation of total colour changes $\left(\Delta \mathrm{E}^{*}\right)$ by Kruskal-Wallis test - tangential surface.

\begin{tabular}{|c|c|c|c|c|c|c|c|c|c|c|}
\hline \multicolumn{11}{|c|}{ Final evaluation of colour - total colour change $\left(\Delta \mathrm{E}^{*}\right)$} \\
\hline \multicolumn{5}{|c|}{ Kruskal-Wallis test } & \multicolumn{6}{|c|}{ TANGENTIAL SURFACE } \\
\hline \multicolumn{4}{|c|}{ Established difference of data } & YES & & & & & & \\
\hline \multirow[t]{2}{*}{ Multiple comparisons } & & & & & & & & & & \\
\hline & 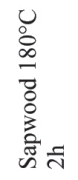 & 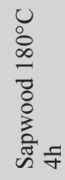 & 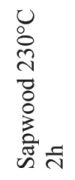 & 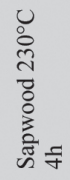 & 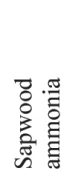 & 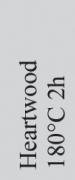 & 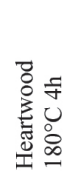 & 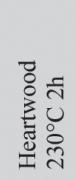 & 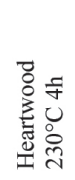 & 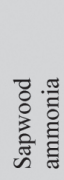 \\
\hline Sapwood $180^{\circ} \mathrm{C} 2 \mathrm{~h}$ & & Agr. & Dif. & Dif. & Agr. & Dif. & Agr. & Dif. & Dif. & Agr. \\
\hline Sapwood $180^{\circ} \mathrm{C} 4 \mathrm{~h}$ & Agr. & & Dif. & Dif. & Agr. & Dif. & Agr. & Dif. & Dif. & Agr. \\
\hline Sapwood $230^{\circ} \mathrm{C} 2 \mathrm{~h}$ & Dif. & Dif. & & Agr. & Dif. & Dif. & Dif. & Agr. & Agr. & Agr. \\
\hline Sapwood $230^{\circ} \mathrm{C} 4 \mathrm{~h}$ & Dif. & Dif. & Agr. & & Dif. & Dif. & Dif. & Agr. & Agr. & Agr. \\
\hline Sapwood ammonia & Agr. & Agr. & Dif. & Dif. & & Dif. & Agr. & Agr. & Dif. & Agr. \\
\hline Heartwood $180^{\circ} \mathrm{C} 2 \mathrm{~h}$ & Dif. & Dif. & Dif. & Dif. & Dif. & & Agr. & Dif. & Dif. & Dif. \\
\hline Heartwood $180^{\circ} \mathrm{C} 4 \mathrm{~h}$ & Agr. & Agr. & Dif. & Dif. & Agr. & Agr. & & Dif. & Dif. & Dif. \\
\hline Heartwood $230^{\circ} \mathrm{C} 2 \mathrm{~h}$ & Dif. & Dif. & Agr. & Agr. & Agr. & Dif. & Dif. & & Agr. & Agr. \\
\hline Heartwood $230^{\circ} \mathrm{C} 4 \mathrm{~h}$ & Dif. & Dif. & Agr. & Agr. & Dif. & Dif. & Dif. & Agr. & & Agr. \\
\hline Heartwood ammonia & Agr. & Agr. & Agr. & Agr. & Agr. & Dif. & Dif. & Agr. & Agr. & \\
\hline
\end{tabular}

Figure 4 shows that when the same treatment was used (treatment temperature and time), the colour of sap- and heartwood was changed almost equally. Due heat treatment the total colour changes 25-40 units are comparable with work from Bekhta and Niemz (2003). Exception was noticed for treatment by ammonia. As can be seen, the total colour change $\left(\Delta \mathrm{E}^{*}\right)$ of sap- and heartwood was different by more than about 7 units. $\Delta \mathrm{E}^{*}$ was due ammonia treatment changed about 17-30 units, similar results were presented by Weigl et al. (2009a, 2012), which mentioned values about 24 units for oak wood. The results are comparable with the results presented by Tinkler (1921).

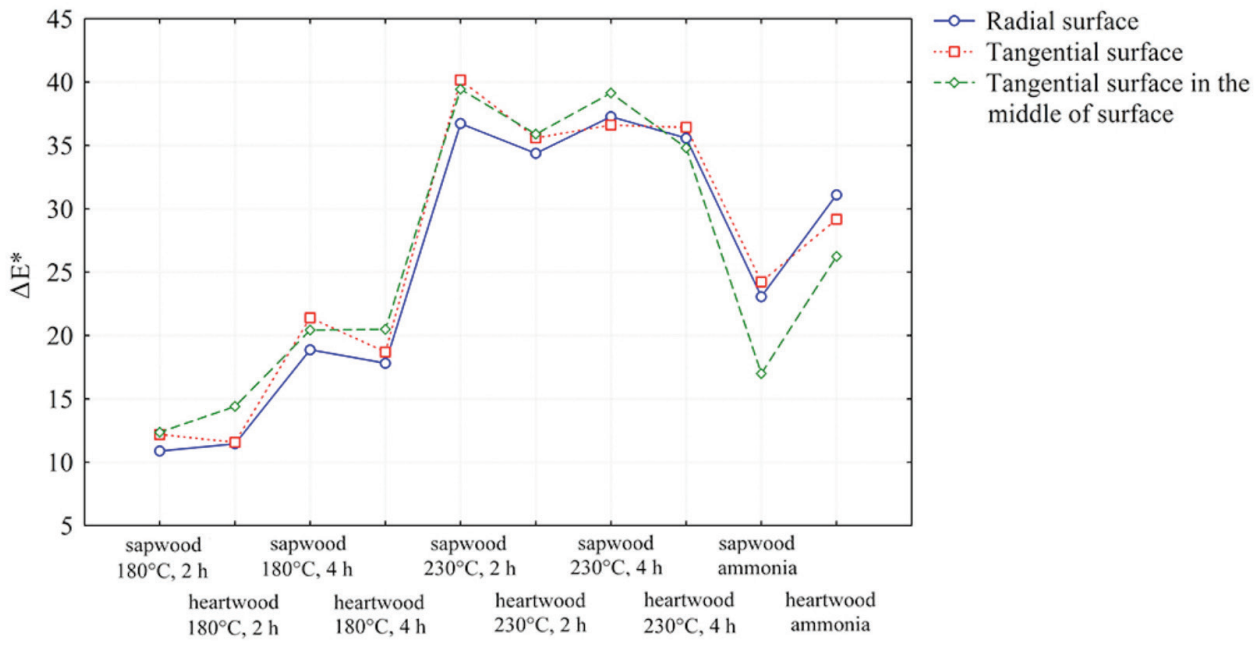

Figure 4. Changes of total colour $\left(\Delta \mathrm{E}^{*}\right)$ for all specimens. 


\section{The effect of heat and ammonia treatment on density ( $\rho 12)$}

Table 5 shows median values of Density before treatment and after treatment for all conditions. Variability of density in all groups can be seen in figure 5.

Table 5. Median values of Density for treated and untreated specimens.

\begin{tabular}{c|c|c}
\hline \multicolumn{3}{c}{ Density $\left(\mathrm{kg} \mathrm{m}^{-3}\right)$} \\
\hline & Untreated & Treated \\
\hline Sapwood $180^{\circ} \mathrm{C} 2 \mathrm{~h}$ & 530,49 & 519,65 \\
\hline Sapwood $180^{\circ} \mathrm{C} 4 \mathrm{~h}$ & 605,92 & 580,09 \\
\hline Sapwood $230^{\circ} \mathrm{C} 2 \mathrm{~h}$ & 568,39 & 528,01 \\
\hline Sapwood $230^{\circ} \mathrm{C} 4 \mathrm{~h}$ & 571,46 & 528,78 \\
\hline Sapwood ammonia & 523,73 & 525,13 \\
\hline Heartwood $180^{\circ} \mathrm{C} 2 \mathrm{~h}$ & 612,48 & 589 \\
\hline Heartwood $180^{\circ} \mathrm{C} 4 \mathrm{~h}$ & 602,93 & 583,3 \\
\hline Heartwood $230^{\circ} \mathrm{C} 2 \mathrm{~h}$ & 605,43 & 562,36 \\
\hline Heartwood $230^{\circ} \mathrm{C} 4 \mathrm{~h}$ & 610,48 & 558,78 \\
\hline Heartwood ammonia & 607,96 & 603,39 \\
\hline
\end{tabular}

Figure 5 shows the effect of treatment, temperature and time on density of sap- and heartwood. It has been confirmed that density decreased as treatment temperature and duration increased. The most significant changes were observed at heat treatment at $230^{\circ} \mathrm{C}$ for $4 \mathrm{~h}$. The decrease was by about $42.68 \mathrm{~kg} \mathrm{~m}^{-3}$ for sapwood and about $51.7 \mathrm{~kg} \mathrm{~m}^{-3}$ for heartwood on average. The decrease of density at heat treatment was confirmed by many authors. Boonstra et al. (2007) reported a 10\% and 8.5\% decrease on density for heat-treated Scots pine and Norway spruce, respectively. Similar results were also confirmed by others (Yildiz 2002, Korkut et al. 2008). The influence of ammonia treatment on density can be confirmed as insignificant (Weigl et al. 2012).

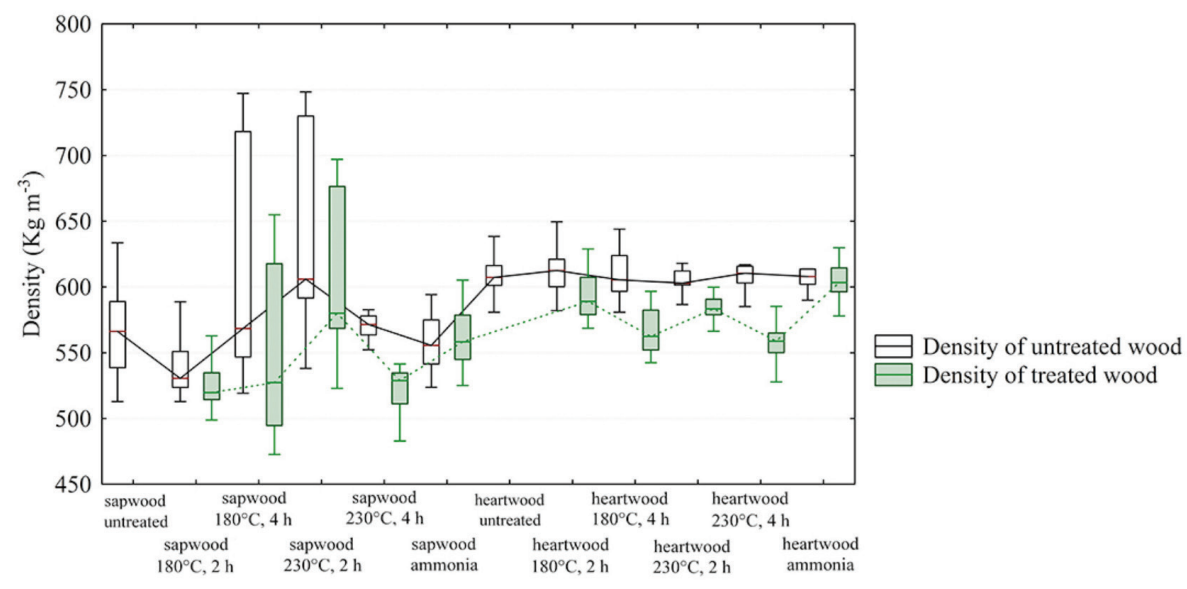

Figure 5. Box plot of density for all treated and untreated specimens.

\section{The effect of heat and ammonia treatment on hardness}

Table 6 shows median values of hardness before treatment and after treatment for all conditions in the radial and tangential direction. Results were recalculated for moisture content $12 \%$. 
Table 6. Median values of Hardness by Janka method for treated and untreated specimens.

\section{Hardness by Janka method (MPa)}

\begin{tabular}{l|c|c|c|c}
\hline \multirow{2}{*}{\multicolumn{2}{c}{ Radial direction }} & \multicolumn{2}{c}{ Tangential direction } \\
\cline { 2 - 5 } & Untreated & Treated & Untreated & Treated \\
\hline Sapwood $180^{\circ} \mathrm{C} 2 \mathrm{~h}$ & 31,47 & 31,31 & 22,58 & 23,93 \\
\hline Sapwood $180^{\circ} \mathrm{C} 4 \mathrm{~h}$ & 36,21 & 36,8 & 30,22 & 31,22 \\
\hline Sapwood $230^{\circ} \mathrm{C} 2 \mathrm{~h}$ & 32,97 & 27,28 & 26,36 & 22,13 \\
\hline Sapwood $230^{\circ} \mathrm{C} 4 \mathrm{~h}$ & 32,54 & 28,33 & 23,49 & 19,77 \\
\hline Sapwood ammonia & 32,36 & 31,6 & 22,98 & 22,8 \\
\hline Heartwood $180^{\circ} \mathrm{C} 2 \mathrm{~h}$ & 35,85 & 36,77 & 26,39 & 26,15 \\
\hline Heartwood $180^{\circ} \mathrm{C} 4 \mathrm{~h}$ & 33,78 & 33,11 & 26,15 & 26,42 \\
\hline Heartwood $230^{\circ} \mathrm{C} 2 \mathrm{~h}$ & 36,07 & 28,82 & 27,05 & 21,76 \\
\hline Heartwood $230^{\circ} \mathrm{C} 4 \mathrm{~h}$ & 34,95 & 30,16 & 27,51 & 22,18 \\
\hline Heartwood ammonia & 34,44 & 38,32 & 28,42 & 29,1 \\
\hline
\end{tabular}

Figure 6 shows the effect of treatment, temperature and time on hardness of sap- and heartwood in the radial and the tangential directions. Minimal changes in the hardness of specimens treated by ammonia and heat at $180^{\circ} \mathrm{C}$ were noticed. The most significant changes were observed at the highest temperature: decrease of 5.3 MPa in the radial and $7.25 \mathrm{MPa}$ in the tangential direction. Hardness of heartwood treated by ammonia increased by about $3.87 \mathrm{MPa}$ in the radial and $0.68 \mathrm{MPa}$ in the tangential direction. A slight increase can be also seen for sapwood at $180^{\circ} \mathrm{C}$ for $4 \mathrm{~h}$ at $0.6 \mathrm{MPa}$ and for heartwood at $180^{\circ} \mathrm{C}$ for $2 \mathrm{~h}$ at $0.92 \mathrm{MPa}$.

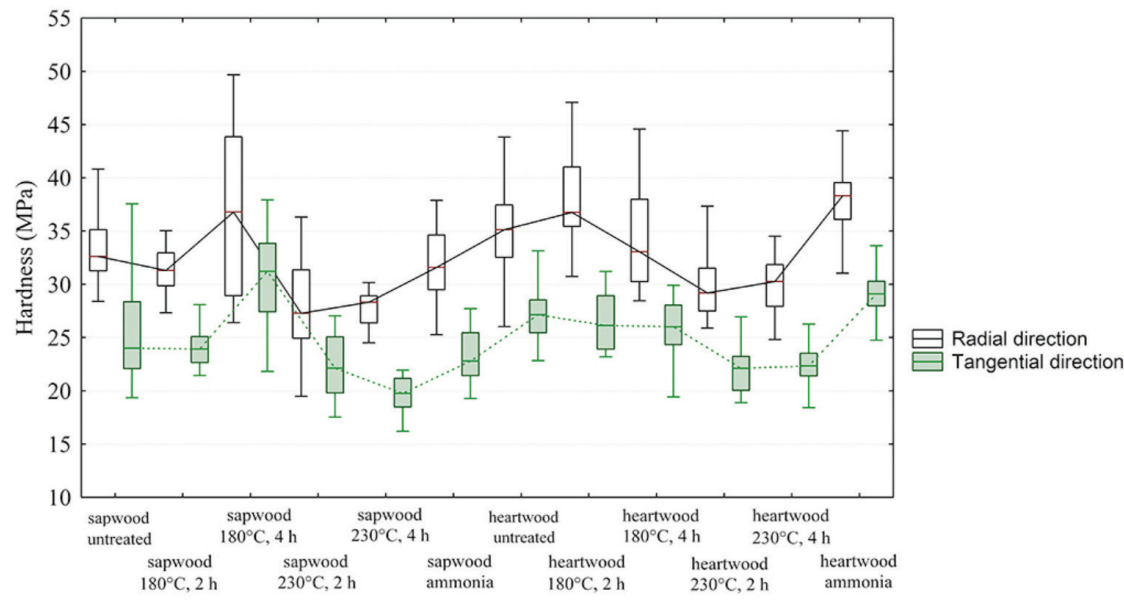

Figure 6. Box plot of hardness in radial and tangential directions for all specimens. 


\section{The effect of heat and ammonia treatment on equilibrium moisture content (EMC)}

Table 7 shows basic statistic values of equilibrium moisture content for all investigated conditions. The last column of table assess whether data came from a normal distribution or not.

Table 7. Basic statistic values of equilibrium moisture content (\%) for treated and untreated specimens.

\begin{tabular}{|c|c|c|c|c|c|c|c|c|c|}
\hline & 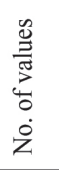 & 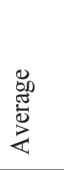 & 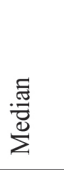 & 声 & 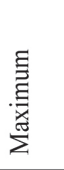 & 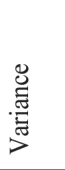 & 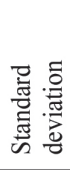 & 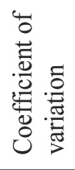 & 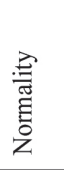 \\
\hline Sapwood untreated & 30 & 7,24 & 7,21 & 6,9 & 7,84 & 0,04 & 0,2 & 2,76 & YES \\
\hline Sapwood $180^{\circ} \mathrm{C} 2 \mathrm{~h}$ & 30 & 3,2 & 3,16 & 2,66 & 3,83 & 0,07 & 0,26 & 7,97 & YES \\
\hline Sapwood $180^{\circ} \mathrm{C} 4 \mathrm{~h}$ & 30 & 3,21 & 3,22 & 2,89 & 3,5 & 0,03 & 0,16 & 5,06 & YES \\
\hline Sapwood $230^{\circ} \mathrm{C} 2 \mathrm{~h}$ & 30 & 2,05 & 2,06 & 1,46 & 2,42 & 0,04 & 0,19 & 9,4 & YES \\
\hline Sapwood $230^{\circ} \mathrm{C} 4 \mathrm{~h}$ & 30 & 2 & 1,98 & 1,84 & 2,22 & 0,01 & 0,1 & 4,93 & YES \\
\hline Sapwood ammonia & 30 & 7,13 & 7,14 & 6,76 & 7,52 & 0,03 & 0,18 & 2,46 & YES \\
\hline Heartwood untreated & 30 & 7,44 & 7,43 & 6,96 & 8,02 & 0,08 & 0,28 & 3,81 & YES \\
\hline Heartwood $180^{\circ} \mathrm{C} 2 \mathrm{~h}$ & 30 & 3,41 & 3,4 & 3,15 & 4,79 & 0,09 & 0,3 & 8,64 & YES \\
\hline Heartwood $180^{\circ} \mathrm{C} 4 \mathrm{~h}$ & 30 & 2,89 & 2,89 & 2,66 & 3,21 & 0,02 & 0,15 & 5,32 & YES \\
\hline Heartwood $230^{\circ} \mathrm{C} 2 \mathrm{~h}$ & 30 & 2,07 & 2,06 & 1,87 & 2,23 & 0,01 & 0,08 & 4,02 & YES \\
\hline Heartwood $230^{\circ} \mathrm{C} 4 \mathrm{~h}$ & 30 & 1,97 & 1,96 & 1,87 & 2,22 & 0,01 & 0,08 & 3,88 & YES \\
\hline Heartwood ammonia & 30 & 6,5 & 6,59 & 3,28 & 6,98 & 0,4 & 0,64 & 9,79 & YES \\
\hline
\end{tabular}

Figure 7 shows the effect of treatment, temperature and time on EMC of sap- and heartwood. The specimens which were conditioned at a temperature of $20^{\circ} \mathrm{C}$ and a relative humidity of $40 \%$ had moisture content of about $7.5 \%$. The treated specimens were compared with these references values. Results show that EMC decreased as the treatment temperature increased. The influence of time seems to be negligible. EMC of treated specimens was more than 50\% lower (1.9-3.2\%). Similar results were shown by Bekhta and Niemz (2003), which mentioned improve of EMC about 30-45\%. Other authors also reported influence of heat treatment on EMC, Tjeerdsma et al. (1998) reported improve about 30-60\%. Ammonia treatment seems to have an insignificant effect on EMC.

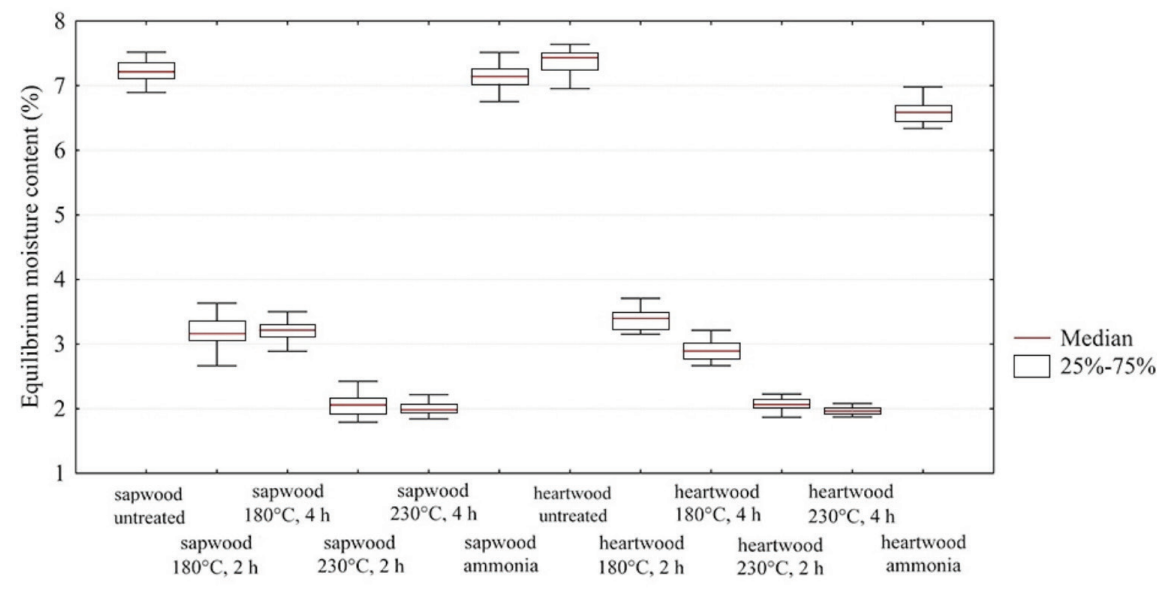

Figure 7. Box plot of equilibrium moisture content (\%) for all specimens. 


\section{The effect of heat and ammonia treatment on modulus of elasticity (MOE) in compression}

Table 8 shows median values of Modulus of elasticity in compression before treatment and after treatment for all conditions in the radial and tangential direction.

Table 8. Median values of Modulus of elasticity in compression for treated and untreated specimens.

\section{Modulus of elasticity in compression (MPa)}

\begin{tabular}{|c|c|c|c|c|}
\hline & $\operatorname{Rac}$ & & Tange & tion \\
\hline & Untreated & Treated & Untreated & Treated \\
\hline Sapwood $180^{\circ} \mathrm{C} 2 \mathrm{~h}$ & 977,51 & 898,14 & 650,08 & 705,89 \\
\hline Sapwood $180^{\circ} \mathrm{C} 4 \mathrm{~h}$ & 1155,86 & 1017,61 & 921,6 & 884,17 \\
\hline Sapwood $230^{\circ} \mathrm{C} 2 \mathrm{~h}$ & 1044,92 & 821,91 & 783,11 & 612,32 \\
\hline Sapwood $230^{\circ} \mathrm{C} 4 \mathrm{~h}$ & 1011,88 & 971,14 & 670,89 & 564,1 \\
\hline Sapwood ammonia & 930,07 & 953,75 & 671,35 & 683,6 \\
\hline Heartwood $180^{\circ} \mathrm{C} 2 \mathrm{~h}$ & 1075,2 & 1171,33 & 757,92 & 752,65 \\
\hline Heartwood $180^{\circ} \mathrm{C} 4 \mathrm{~h}$ & 1031,53 & 1131,4 & 791,82 & 757,37 \\
\hline Heartwood $230^{\circ} \mathrm{C} 2 \mathrm{~h}$ & 1161,17 & 983,34 & 795,99 & 616,21 \\
\hline Heartwood $230^{\circ} \mathrm{C} 4 \mathrm{~h}$ & 1070,21 & 895,17 & 811,79 & 635,9 \\
\hline Heartwood ammonia & 999,42 & 1203,02 & 833,08 & 852,36 \\
\hline
\end{tabular}

Figure 8 shows the effect of treatment, temperature and time on modulus of elasticity in compression of sapand heartwood in the radial and the tangential directions. No important changes in MOE have been proved. The most significant changes can be seen for sapwood at a temperature of $230^{\circ} \mathrm{C}$ : a decrease of about $233 \mathrm{MPa}$ in the radial direction and $170.78 \mathrm{MPa}$ in the tangential direction. For heartwood a decrease on average $177 \mathrm{MPa}$ can be noticed. Interesting changes can be noticed concerning the specimens which were treated by ammonia: an increase in MOE for heartwood of about 203.61 MPa in the radial direction and 19.28 MPa in the tangential direction. 


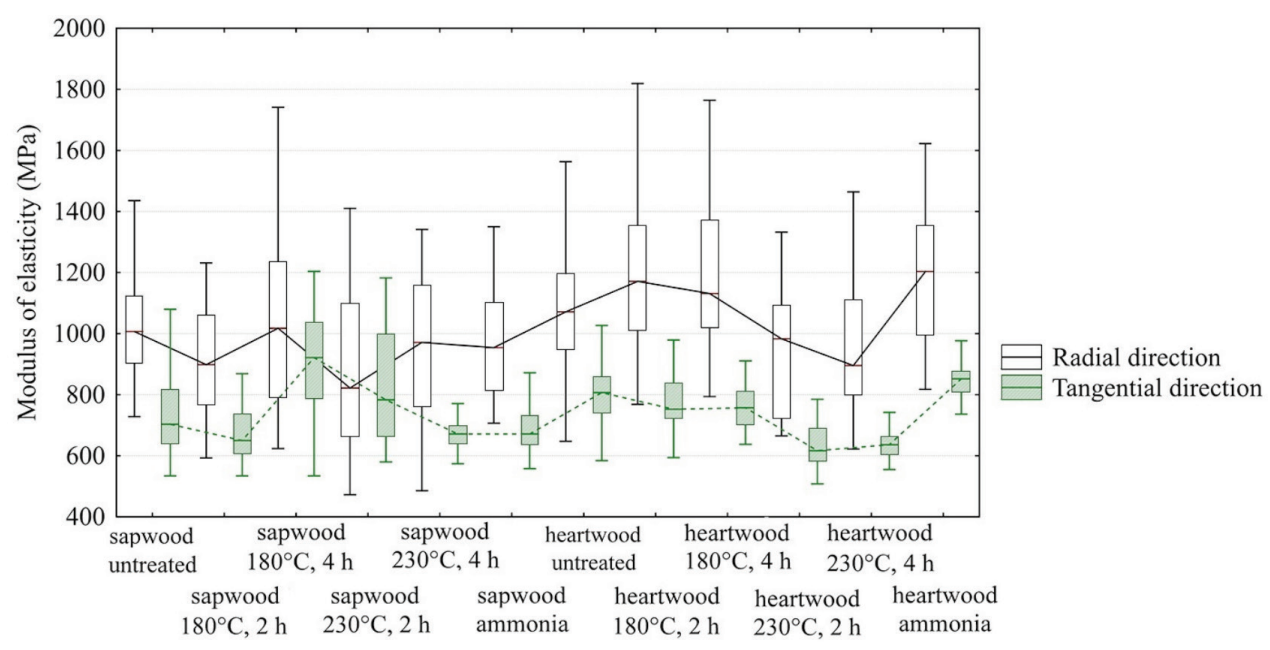

Figure 8. Box plot of modulus of elasticity in radial and tangential directions for all specimens.

Even that used samples were quite small $(30 \times 30 \times 30 \mathrm{~mm})$, results or methods can be partially useful for practical application, but another studies of influence of both treatment on demanded properties should be done. Ammonia treatment was in last decades used for plasticizing of wood - Lignamon material (Rosca et al. 2002, Bariska 1969), but currently is partly replaced by other modification methods - heat treatment, chemical modification etc. (Hill 2006)

\section{CONCLUSION}

The influence of heat and ammonia treatment on colour response of oak wood was studied and physical and mechanical properties were compared. The heat and ammonia treatments have a significant influence on colour change. Differences of total colour changes $\left(\Delta \mathrm{E}^{*}\right)$ between these processes for sap-and heartwood were observed. The most significant total colour changes between sap- and heartwood were found where comparing specimens treated by ammonia. Ammonia treatment may significantly improve the decorative value of wood. However, the ammonia treatment is a soft modification with respect to physical and mechanical properties. Heat treatment shows the smallest changes in colour at $180^{\circ} \mathrm{C}$ for $2 \mathrm{~h}$. Then the specimens become darker with increasing treatment time and temperature. However, heat treatment caused a decrease in the values of investigated physical and mechanical properties (EMC, density, MOE and hardness). In this respect, the heat and ammonia treatment are comparable processes for colour change.

\section{ACKNOWLEDGEMENT}

This work was funded by the European Social Fund and the state budget of the Czech Republic, project "The Establishment of an International Research Team for the Development of New Wood-based Materials" reg. no. CZ.1.07/2.3.00/20.0269 (EE2.3.20.0269). This study was also supported by the Internal Grant Agency (IGA) of Faculty of Forestry and Wood Technology, Mendel University in Brno - project no. 26/2012 and IGA-V 50/2011. 


\section{REFERENCES}

Baar, J.; Gryc, V. 2012. The analysis of tropical wood discoloration caused by simulated sunlight. European Journal of Wood and Wood Products 70: 263-269.

Bariska, M. 1969. Plastifizierung des Holzes mit Ammoniak in Theorie und Praxis. Holz-Zentralblatt 95(84):1309-1311.

Bekhta, P.; Niemz, P. 2003. Effect of high temperature on the change in color, dimensional Stability and mechanical properties of spruce wood. Holzforschung 57: 539-546.

Boonstra, M.; Van Acker, J.; Tjeerdsma, B.; Kegel, E. 2007. Strength properties of thermally modified softwoods and its relation to polymeric structural wood constituents. Ann Forest Sci 64: 679-690.

Brischke, C.; Welzbacher, C.; Brandt, K.; Rapp, A. 2007. Quality control of thermally modified timber: Interrelationship between heat treatment intensities and CIE L*a*b* color data on homogenized wood samples. Holzforschung 61: 19-22.

Esteves, B.; Velez Marques, A.; Domingos, I.; Pereira, H. 2008. Heat induced colour changes of pine (Pinus pinaster) and eucalypt (Eucalyptus globulus) wood. Wood Sci Technol 42(5) 369-384.

Hill, C. 2006. Wood Modification-Chemical, Thermal and Other Processes, Wiley Series in Renewable Resources, John Wiley \& Sons, Ldt.

Jämsä, S.; Viitaniemi, P. 2001. Heat treatment of wood - Better durability without chemicals, In: Proceedings of special seminar held in Antibes, France.

Korkut, S.; Kök, M.; Korkut, D.; Gürleyen T. 2008. The effects of heat treatment on technological properties in Red-bud maple (Acer trautvetteri Medw.) wood. Bioresour Technol 99:1538-1543.

McDonald, A.; Fernandez, M.; Kreber, B. 1997. Chemical and UV-VIS spectroscopic study on kiln brown stain formation in radiata pine. In: $9^{\text {th }}$ International Symposium of Wood and Pulping Chemistry, Montreal, Canada $70,1-5$.

Militz, H. 2002. Heat treatment of wood: European processes and thein background. In: International Research Group Wood Pre, Section 4-Processes, No IRG/WP 02-40241.

Mitsui, K.; Takada, H.; Sugiyama, M.; Hasegawa, R. 2001. Changes in the properties of light irradiated wood with heat treatment. Part 1. Effect of treatment conditions on the change in color. Holzforschung 55: 601-605.

Mitsui, K.; Murata, A.; Tolvaj, L. 2004. Changes in the properties of lightirradiated wood with heat treatment: Part 3. Monitoring by DRIFT spectroscopy. Holz Roh-Werkst 62: 164-168.

Mitsui, K.; Murata, A.; Kohara, M.; Tsuchikawa, S. 2003. Colour modification of wood by light-irradiation and heat treatment. In: Abstracts of the First European Conference on Wood Modification, Belgium.

Rosca, I.; Pühringer, R.; Schmidt, H.; Tanczos, I. 2002. New aspects in studying and applications of ammonia treatment of softwood. Proceedings of the $4^{\text {th }}$ IUFRO Symposium. 1-3 of September, Bystrá, Slovakia.

Sehlstedt-Persson, M. 2003. Colour responses to heat treatment of extractives and sap from pine and spruce. $8^{\text {th }}$ International IUFRO Wood Drying Conference, Brasov, Romania, 459-464. 
Siau, J.F. 1984. Transport Processes in Wood. Springer-Verlag, Berlin, Heidelberg, New York.

Stamm, A.; Burr, H.; Kline, A. 1946. Stay-wood-A heat stabilized wood. Ind Eng Chem 38(6): 630-634.

Sundqvist, B. 2004. Colour changes and acid formation in wood during heating. Doctoral Thesis. Lulea University of Technology. Skelleftea, Sweden. 154p.

Sundqvist, B.; Morén, T. 2002. The influence of wood polymers and extractives on wood colour induced by hydrothermal treatment. Holz Roh-Werkst 60: 375-376.

Tiemann, H. 1920. Effect of Different Methods of Drying on the Strength and Hygroscopicity of Wood. $3^{\text {rd }}$ Ed. The kiln drying of lumber Chap. 11, J.P. Lippincott Co.

Tjeerdsma, B.; Boonstra, M.; Militz, H. 1998. Thermal modification of nondurable wood species. Part 2. Improved wood properties of thermally treated wood. International Research Group on Wood Pre., Document no. IRG/WP 98- 40124.

Tinkler, C. K. 1921. Fumed oak and natural brown oak. Biochem J 15(4): 477-486.

Vavrčík, H.; Gryc, V. 2012. Analysis of the annual ring structure and wood density relations in English oak and Sessile oak. Wood Research 57 (4): 573-580.

Vavrčík, H.; Gryc, V.; Rybníček, M. 2008. Variability of selected physical and mechanical properties of English oak wood. Influence of age, trunk radius and height. Lesnická práce, s.r.o., Kostelec nad Černými lesy [in Czech].

Weigl, M.; Gündüz, M.; Müller, U. 2009a. On the mechanical stability of ammonia treated wood. In: Hill CAS, Militz H (eds) $4^{\text {th }}$ European Conference on Wood Modification. 27 $7^{\text {th }}-29^{\text {th }}$ April 2009, Stockholm, Sweden.

Weigl, M.; Kandelbauer, A.; Hansmann, C.; Pöckl, J.; Müller, U.; Grabner, M. 2009b. Application of Natural Dyes in the Coloration of Wood. In: Bechtold T, Mussak R (eds) Handbook of natural colorants. Wiley, New York, pp 277-313.

Weigl, M.; Müller, U.; Wimmer, R.; Hansmann, C. 2012. Ammonia vs. thermally modified timber-comparison of physical and mechanical properties. European Journal of Wood and Wood Products 70(1-3): 233-239.

Weigl, M.; Pöckl, J.; Grabner, M. 2009c. Selected properties of gas phase ammonia treated wood. European Journal of Wood and Wood Products 67:103-109.

Weigl, M.; Pöckl, J.; Müller, U.; Pretzl, H.; Grabner, M. 2007. UV resistance of ammonia treated wood. In: Hill CAS, Jones D, Militz H, Ormondroyd GA (eds). 3rd European Conference on Wood Modification. $15^{\text {th }}-16^{\text {th }}$ October 2007, Cardiff, UK.

Yildiz, S. 2002. Physical, mechanical, technological and chemical properties of beech and spruce wood treated by heating. PhD dissertation, Karadeniz Tech. Univ., Trabzon, Turkey. 200p. 
